\title{
Exophiala jeanselmei
}

National Cancer Institute

\section{Source}

National Cancer Institute. Exophiala jeanselmei. NCI Thesaurus. Code C127710.

A species of saprobic fungi in the phylum Ascomycota. E. jeanselmei is the causative agent of maduromycosis, an asymptomatic disease which presents with black or brown macular lesions. 